\title{
Impedance description of coherent synchrotron radiation with account of bunch deformation
}

\author{
Robert Warnock* and Ronald Ruth ${ }^{\dagger}$ \\ Stanford Linear Accelerator Center, Stanford University, Stanford, California 94309, USA \\ Marco Venturini ${ }^{\ddagger}$ \\ Lawrence Berkeley National Laboratory, University of California, Berkeley, California, 94720, USA \\ James A. Ellison ${ }^{\S}$ \\ Department of Mathematics and Statistics, University of New Mexico, Albuquerque, New Mexico, 87131, USA
}

(Received 21 October 2004; published 28 January 2005)

\begin{abstract}
We are concerned with coherent longitudinal motion in a storage ring, especially with situations in which coherent synchrotron radiation (CSR) can influence stability of the beam. The collective force from CSR is usually described by an impedance or a wake function in such a way that the force depends only on the charge distribution at the present time. This description is exact only for a rigid bunch, since causality demands that the force depend on the prior history of the bunch. We show how to treat a deforming bunch by applying the "complete impedance" $Z(n, \omega)$, a function of wave number and frequency. We derive this impedance and study its analytic properties for a special model: radiation from circular orbits shielded by parallel plates representing the metallic vacuum chamber. We analyze the corresponding collective force, obtaining the usual formula as a first approximation, plus easily computed corrections that depend on present and prior values of the time derivative of the charge density. In related papers we have applied these results in numerical simulations of instabilities induced by CSR.
\end{abstract}

DOI: $10.1103 /$ PhysRevSTAB.8.014402

PACS numbers: 29.27.Bd, 41.60.Ap

\section{INTRODUCTION}

The concept of impedance is often invoked to discuss beam stability in storage rings [1], for both longitudinal and transverse motion. The longitudinal impedance, which is presently our main object of interest, specifies the longitudinal collective force acting on the beam itself, in a particular electromagnetic environment. Consequently, it specifies a feedback mechanism that can lead to instabilities at sufficiently high current. One often thinks of the impedance as primarily a property of the environment, namely, the metallic vacuum chamber surrounding the beam, but it can also include effects of space charge and trajectory curvature that are present even in the absence of a vacuum chamber.

The idea of impedance, at least in its usual elementary form, has significant limitations. It is useful primarily when the transverse extent of the beam can be neglected. Even in that case it does not give the exact form of the wake field unless the charge distribution is rigid (independent of time in the beam frame). To express the collective force on an evolving distribution the common practice is to adopt the formula for the rigid case, merely replacing the rigid distribution by the evolving one evaluated at the present time. This is not correct in principle, since causality and the finite velocity of light demand that the present field depend

\footnotetext{
*Electronic address: warnock@slac.stanford.edu

${ }^{\dagger}$ Electronic address: rruth@slac.stanford.edu

Electronic address: mventurini@lbl.gov

${ }^{\S}$ Electronic address: ellison@math.unm.edu
}

on prior values of its source. This is obvious in the case of particles following a curved trajectory, since a wave emitted from a bunch at a particular time can catch up with the bunch at a later time when the bunch has an altered form.

In this paper we extend the scope of the impedance description to account for retardation with bunch deformation. This requires a function $Z(n, \omega)$ of two variables (wave number and frequency). We call this function the complete impedance, to distinguish it from the impedance as conventionally defined which is $Z(n)=Z\left(n, n \omega_{0}\right)$, where $\omega_{0}$ is the angular revolution frequency. We call $Z(n)$ the elementary impedance. We derive and analyze the complete impedance only for a special model, the case of radiation from circular orbits with a parallel plate model of the vacuum chamber. The analysis points to certain features that can be expected in a more general setting, but also features that must be quite peculiar to the model. At present we cannot offer a model-independent version of the extended impedance picture, but we expect that other explicitly soluble models can be analyzed along the lines of the present study.

The collective force as expressed in terms of $Z(n, \omega)$ involves a sum on $n$ and integrals on $\omega$ and the time $t$, and is therefore expensive to evaluate numerically. Fortunately, one can find systematic approximations to the force that are easy to compute.

Much of the present analysis was motivated by our work on numerical simulation of many-particle longitudinal dynamics in the presence of coherent synchrotron radiation (CSR) [2-4]. Accordingly, the viewpoint of the following is that the evolving charge density is not a given function, 
but is determined dynamically in a self-consistent manner. For that we carried out a time-domain integration of the nonlinear Vlasov or Vlasov-Fokker-Planck equation, but macroparticle simulations could be used as well.

In Sec. II we find the general form of the longitudinal collective force, and also the radiated power, in terms of the complete impedance and the time-dependent Fourier transform of the charge distribution. The expression for the force must be evaluated anew at each time step in a selfconsistent simulation. Since an evaluation of the exact formula would be too expensive, it is essential to find good approximations that are easier to compute. In Sec. III we derive a systematic sequence of approximations. At lowest order we find the simple adaptation of the rigid bunch formula mentioned above, which was employed in Refs. [2,3]. The first correction, which was explored numerically in [4], involves present and prior values of the time derivative of the charge density. The work of Secs. II and III depends on detailed analytic properties of the complete impedance, which are determined in Sec. IV. We find both the longitudinal and transverse forces, although it is usually believed that the latter have minor dynamical importance. Section V gives a summary and the outlook for further work.

\section{COLLECTIVE FORCE IN TERMS OF THE COMPLETE IMPEDANCE}

We work in cylindrical coordinates $(r, \theta, y)$, with the $y$ axis perpendicular to perfectly conducting infinite plates located at $y= \pm g, h=2 g$. We suppose that the charge/ current distribution has the form of a "vertical ribbon beam." In the bunch frame the line density is $\lambda(\theta, t)$, and in the laboratory frame the normalized particle density $\rho$ and current density $\mathbf{J}$ are as follows:

$$
\begin{aligned}
\rho(r, \theta, y, t) & =\lambda\left(\theta-\omega_{0} t, t\right) \frac{\delta(r-R)}{R} H(y), \\
\mathbf{J} & =\left(J_{r}, J_{\theta}, J_{y}\right)=\left(0, Q \beta_{0} c \rho, 0\right), \\
\int_{0}^{2 \pi} \lambda(\theta, t) d \theta & =1, \quad \lambda(\theta+2 \pi, t)=\lambda(\theta, t), \\
\int_{-g}^{g} d y H(y) & =1,
\end{aligned}
$$

where $Q=\mp e N$ is the total charge and $\omega_{0}=\beta_{0} c / R$ is the angular velocity.

We shall define the impedance in terms of the mean value of the longitudinal electric field with respect to the transverse distribution:

$$
\begin{aligned}
\mathcal{E}(\theta, t) & =\int_{0}^{\infty} r d r \int_{-g}^{g} d y \frac{\delta(r-R)}{R} H(y) E_{\theta}(r, \theta, y, t) \\
& =\int_{-g}^{g} E_{\theta}(R, \theta, y, t) H(y) d y .
\end{aligned}
$$

By (1) the beam current is

$$
I(\theta, t)=\int_{0}^{\infty} d r \int_{-g}^{g} d y J_{\theta}(r, \theta, y, t)=Q \omega_{0} \lambda\left(\theta-\omega_{0} t, t\right),
$$

which has the formal Fourier transform

$$
\begin{aligned}
\hat{I}(n, \omega) & =\frac{1}{(2 \pi)^{2}} \int_{-\infty}^{\infty} d t e^{i \omega t} \int_{0}^{2 \pi} d \theta e^{-i n \theta} I(\theta, t) \\
& =\frac{Q \omega_{0}}{2 \pi} \int_{-\infty}^{\infty} d t e^{i\left(\omega-n \omega_{0}\right) t} \lambda_{n}(t),
\end{aligned}
$$

where

$$
\lambda_{n}(t)=\frac{1}{2 \pi} \int_{0}^{2 \pi} d \theta e^{-i n \theta} \lambda(\theta, t) .
$$

Depending on the model of $\lambda_{n}(t)$, the formal transform (4) is not necessarily a proper Fourier transform. For instance it may contain a delta-function component, as in the idealized case of a rigid bunch with $\lambda_{n}(t)=\lambda_{n}=$ const. In that case we have

$$
\hat{I}(n, \omega)=Q \omega_{0} \lambda_{n} \delta\left(\omega-n \omega_{0}\right) .
$$

To allow a deforming bunch we apply the Laplace transform rather than the Fourier transform, redefining $\hat{I}$ to be

$$
\hat{I}(n, \omega)=\frac{Q \omega_{0}}{2 \pi} \int_{0}^{\infty} e^{i\left(\omega-n \omega_{0}\right) t} \lambda_{n}(t) d t, \quad \operatorname{Im} \omega>0 .
$$

To maintain contact with the familiar notation of Fourier analysis, we use $\omega$ rather than the conventional Laplace variable $s=-i \omega$ as the variable conjugate to $t$.

To be precise we assume physically reasonable conditions on $\lambda_{n}(t)$ for $t>0$, namely, that it has a continuous second derivative and that $\left|\lambda_{n}\right|,\left|\lambda_{n}^{\prime}\right|,\left|\lambda_{n}^{\prime \prime}\right|$ are each bounded. Then with $\operatorname{Im} \omega=v>0$ the following Laplace transforms exist:

$$
\begin{gathered}
\hat{\lambda}_{n}(\omega)=\frac{1}{2 \pi} \int_{0}^{\infty} e^{i \omega t} \lambda_{n}(t) d t \\
\hat{\lambda}_{n}^{\prime}(\omega)=\frac{1}{2 \pi} \int_{0}^{\infty} e^{i \omega t} \lambda_{n}^{\prime}(t) d t=-i \omega \hat{\lambda}_{n}(\omega)-\frac{1}{2 \pi} \lambda_{n}(0) .
\end{gathered}
$$

Since $\lambda_{n}$ and $\lambda_{n}^{\prime}$ are both smooth and bounded, the inversion theorem [5] guarantees that

$$
\lambda_{n}(t)=\lim _{U \rightarrow \infty} \int_{-U+i v}^{U+i v} e^{-i \omega t} \hat{\lambda}_{n}(\omega) d \omega, \quad t>0,
$$

and similarly for $\lambda_{n}^{\prime}(t)$. The improper integral defined as a symmetric limit in (10) is often called the "principal value." It exists under weaker conditions than are required for existence of separate integrals over the two half-lines $(-\infty+i v, i v),(i v, \infty+i v)$. We shall understand all $\omega$ integrals to be principal values, without a notational indication. 
Correspondingly, we take the Laplace transform of Maxwell's equations with respect to $t$. The transform of terms involving time derivatives of fields will produce terms from the values of those fields at $t=0$, as in (9). We set those initial values to zero, since we are interested only in two problems: (i) the initial value problem to be discussed below, in which the beam current and the field are zero for $t \leq 0$; and (ii) the question of stability of an equilibrium state under a small perturbation of the charge distribution, the latter being zero until $t=0$. In problem (ii) we are concerned only with the additional field produced by the perturbation, which is zero for $t \leq$ 0 . The field of the beam in equilibrium enters only in the determination of the equilibrium state.

Defining $\hat{\mathcal{E}}(n, \omega)$ to be the double transform of $\mathcal{E}(\theta, t)$ (Fourier in $\theta$, Laplace in $t$ ), we then find through solution of Maxwell's equations that $\hat{\mathcal{E}}$ is proportional to $\hat{I}$; see Sec. IV. This proportionality defines the impedance $Z$ :

$$
-2 \pi R \hat{\mathcal{E}}(n, \omega)=Z(n, \omega) \hat{I}(n, \omega) .
$$

This does not imply that $Z(n, \omega)$ is itself a proper FourierLaplace transform of a continuous function. An expression for $Z(n, \omega)$ is given in Eq. (63).

From (11) and (7), and the inversion theorems for Laplace and Fourier transforms, we find the induced voltage $V$ as

$$
\begin{aligned}
V(\theta, t) & =-2 \pi R \mathcal{E}(\theta, t) \\
& =\omega_{0} Q \sum_{n} e^{i n \theta} \int_{\operatorname{Im} \omega=v} e^{-i \omega t} Z(n, \omega) \hat{\lambda}_{n}\left(\omega-n \omega_{0}\right) d \omega .
\end{aligned}
$$

Except for a constant factor, $V$ is the collective force that appears in the Vlasov equation; see Eqs. (12) and (20) of [2]. To retrieve the case of a rigid bunch we take $v=0$ and put (6) in place of (7) in (12) to obtain

$$
V(\theta, t)=\omega_{0} Q \sum_{n} e^{i n\left(\theta-\omega_{0} t\right)} Z\left(n, n \omega_{0}\right) \lambda_{n} .
$$

The quantity $Z(n)=Z\left(n, n \omega_{0}\right)$ is what is usually called the impedance [1]. It is not entirely adequate to describe the time-dependent case with evolving bunch form. We need instead the function of two variables $Z(n, \omega)$, wave number $(n / R)$ and frequency, which we shall call the complete impedance. One might conjecture, however, that a first approximation to the collective force would be obtained merely by replacing $\lambda_{n}$ by $\lambda_{n}(t)$ in (13), thus

$$
V(\theta, t) \approx \omega_{0} Q \sum_{n} e^{i n\left(\theta-\omega_{0} t\right)} Z\left(n, n \omega_{0}\right) \lambda_{n}(t) .
$$

We shall in fact derive this approximation, and corrections to it as well, in Sec. III. This amounts to saying that the force at time $t$ is calculated as though the bunch form at time $t-\Delta t$ had held constant at all earlier times. Clearly, retardation effects are not treated exactly.

The formula (12) seems to involve $\lambda_{n}\left(t^{\prime}\right)$ for $t^{\prime}>t$, which would mean a violation of causality. To show that such contributions in fact drop out, we invoke the fact that $Z(n, \omega)$ is analytic and bounded as a function of $\omega$ for $\operatorname{Im} \omega>\epsilon$, any $\epsilon>0$. This and other required properties of $Z$ are proved in Sec. IV. Writing $\Omega=\omega-n \omega_{0}$, we first integrate twice by parts on $t^{\prime}$ so that (8) takes the form

$$
\begin{aligned}
\hat{\lambda}_{n}(\Omega)= & \frac{1}{2 \pi i \Omega}\left[-\lambda_{n}(0)+\frac{\lambda_{n}^{\prime}(0)}{i \Omega}\right. \\
& \left.+\frac{1}{i \Omega}\left(\int_{0}^{t}+\int_{t}^{\infty}\right) e^{i \Omega t^{\prime}} \lambda_{n}^{\prime \prime}\left(t^{\prime}\right) d t^{\prime}\right] .
\end{aligned}
$$

Now it is seen that the term in the $\omega$ integrand from $\int_{t}^{\infty}$ contributes nothing to (12). It is analytic for $\operatorname{Im} \omega>\epsilon>0$ and $\mathcal{O}\left(|\omega|^{-2}\right)$, the latter because exponential increase of $e^{-i \omega t}$ is compensated by exponential decrease of the $\int_{t}^{\infty}$. We can push the contour to a semicircle at infinity, getting zero in the limit.

Now define $\tilde{\lambda}_{n}(\Omega, t)$ as (15) minus its final term which we have just discarded. Convergence of the $\omega$ integral of the first term in $\tilde{\lambda}$ follows from the asymptotic behavior of $Z(n, \omega)$ stated in (70); i.e., since $Z$ tends to a constant (plus an oscillating term) the integral converges by virtue of oscillations of the factor $e^{-i \omega t}$. The remaining $\omega$ integrals converge absolutely.

Undoing the partial integrations that led to (15), we of course get rid of boundary terms at $t^{\prime}=0$ but acquire boundary terms at $t^{\prime}=t$ :

$$
\begin{aligned}
\tilde{\lambda}_{n}(\Omega, t)= & \frac{1}{2 \pi}\left[-\frac{\lambda_{n}^{\prime}(t) e^{i \Omega t}}{\Omega^{2}}+\frac{i \lambda_{n}(t) e^{i \Omega t}}{\Omega}\right. \\
& \left.+\int_{0}^{t} e^{i \Omega t^{\prime}} \lambda_{n}\left(t^{\prime}\right) d t^{\prime}\right] .
\end{aligned}
$$

When (16) is used in (12), the $\omega$ integral of its first term is seen to be zero, again by analyticity and decay as $|\omega|^{-2}$. The resulting expression of $V$ is

$$
\begin{aligned}
V(\theta, t)= & \omega_{0} Q \sum_{n} e^{i n \theta} \int_{\operatorname{Im} \omega=v} d \omega Z(n, \omega) \\
& \times \frac{1}{2 \pi}\left[i \lambda_{n}(t) \frac{e^{-i n \omega_{0} t}}{\omega-n \omega_{0}}\right. \\
& \left.+e^{-i \omega t} \int_{0}^{t} d t^{\prime} e^{i\left(\omega-n \omega_{0}\right) t^{\prime}} \lambda_{n}\left(t^{\prime}\right)\right] .
\end{aligned}
$$

The $\omega$ integral of the first term in the square bracket exists only by virtue of the integral's definition as the principal value. The same is true of the integral of the second term.

We can take the limit as $v \rightarrow 0$ in (17) by applying the usual rule for pushing a contour of integration against a pole. Actually, there are other poles on the real axis to be accounted for, since $Z(n, \omega)$ has poles as a function of $\omega$ at the waveguide cutoffs. That matter is discussed in Sec. IV. 
In Sec. III we shall derive approximations to $V(\theta, t)$ which are more economical to evaluate than (17). For that we make stronger assumptions on smoothness of $\lambda_{n}(t)$, namely, that for $m \geq 0$ it has $m+2$ continuous and bounded derivatives, with derivatives up to order $m+$ 1 vanishing at $t=0$ :

$$
\begin{aligned}
\lambda_{n} & \in C^{m+2}, \quad\left|\lambda_{n}^{(k)}(t)\right| \leq M, \quad k=0,1, \ldots, m+2, \\
\lambda_{n}^{(k)}(0) & =0, \quad k=0,1, \ldots, m+1 .
\end{aligned}
$$

Thus we switch on the current with a certain degree of smoothness at $t=0$. One can think of the switching on as a model of the beam injection process in a storage ring. Since we make no restriction on the magnitude of derivatives for $t>0$, any profile of current building up from zero, even a step function, can be approximated by a function satisfying our conditions. With assumptions (18) we can integrate by parts as many as $m+2$ times in (8), and discard $\int_{t}^{\infty}$ by the same argument as before, to cast (12) in the form

$$
\begin{aligned}
V(\theta, t)= & \omega_{0} Q \sum_{n} e^{i n \theta} \int_{\operatorname{Im} \omega=v} e^{-i \omega t} Z(n, \omega) \\
& \times \mu_{n k}\left(\omega-n \omega_{0}, t\right) d \omega, \\
\mu_{n k}(\Omega, t)= & \frac{1}{2 \pi(-i \Omega)^{k+2}} \int_{0}^{t} e^{i \Omega t^{\prime}} \lambda_{n}^{(k+2)}\left(t^{\prime}\right) d t^{\prime},
\end{aligned}
$$

for any $k \in\{0,1, \ldots, m\}$. In Sec. III we shall use (19) to derive approximations to $V$.

To compute the instantaneous radiated power, note that the work done by the field $\mathcal{E}$ on a charge element $d Q$ when the charge moves a distance $R d \theta=R \omega_{0} d t$ is equal to $d W=d Q \mathcal{E}(\theta, t) R \omega_{0} d t$. The radiated power for the charge element is the corresponding rate of change of field energy, $-d W / d t$. Putting $d Q=Q \lambda\left(\theta-\omega_{0} t, t\right) d \theta$ and integrating over $\theta$ we find the total radiated power, from all charge elements. From (11) that is

$$
\begin{aligned}
\mathcal{P}(t)= & \frac{Q \omega_{0}}{2 \pi} \int d \theta \lambda\left(\theta-\omega_{0} t, t\right) \sum_{n} e^{i n \theta} \\
& \times \int_{\operatorname{Im} \omega=v} e^{-i \omega t} Z(n, \omega) \hat{I}(n, \omega) d \omega \\
= & Q \omega_{0} \sum_{n} e^{i n \omega_{0} t} \lambda_{n}^{*}(t) \int_{\operatorname{Im} \omega=v} e^{-i \omega t} Z(n, \omega) \hat{I}(n, \omega) d \omega .
\end{aligned}
$$

In the case of the rigid bunch one can put $v=0$ and apply (6) to obtain the well-known formula

$$
\mathcal{P}=\left(Q \omega_{0}\right)^{2} \sum_{n} \operatorname{Re} Z\left(n, n \omega_{0}\right)\left|\lambda_{n}\right|^{2}
$$

As in (14), we replace $\lambda_{n}$ by $\lambda_{n}(t)$ in (21) for the approximation to $\mathcal{P}(t)$ used in numerical explorations to date [3]. In general the $\omega$ integral in (20) can be expressed as in (17) or (19). Applying (17) we get

$$
\begin{aligned}
\mathcal{P}(t)= & \frac{\left(Q \omega_{0}\right)^{2}}{2 \pi} \sum_{n} \lambda_{n}^{*}(t) \int_{\operatorname{Im} \omega=v} d \omega Z(n, \omega)\left[\frac{i \lambda_{n}(t)}{\omega-n \omega_{0}}\right. \\
& \left.+\int_{0}^{t} d t^{\prime} e^{i\left(\omega-n \omega_{0}\right)\left(t^{\prime}-t\right)} \lambda_{n}\left(t^{\prime}\right)\right] .
\end{aligned}
$$

\section{APPROXIMATIONS TO THE COLLECTIVE FORCE}

In this section we assume conditions (18) on the particle density. These conditions form the basis for a rational mathematical discussion, but it must be admitted that the conditions are difficult to verify, since in practice $\lambda_{n}(t)$ is determined by a numerical realization of self-consistent dynamics. Nevertheless, an important part of (18), the requirement that time derivatives up to some order be zero initially, can be simulated in a numerical solution of the Vlasov equation. For that we multiply $\lambda_{n}(t)$ by a "smooth ramp" $f \in C^{m+2}$ that is zero for $t<0$, has vanishing derivatives up to order $m+1$ at $t=0$, and is equal to 1 for $t$ greater than some small $t_{0}$. This was done in Ref. [4].

One could, in principle, find the collective force in a time-dependent Vlasov integration by direct numerical evaluations of the two integrals and the sum in (17) or (19). The integral over $t^{\prime}$ would evolve dynamically in steps $\delta t$ of $t$. This would be an expensive algorithm, however, involving evaluation of many negligible contributions. It is expected that only components of the electric field with phase velocity close to the particle velocity will have a big effect on the beam, which is to say that the important part of the $\omega$ integral should lie near $\omega=n \omega_{0}$. To see that mathematically, consider (19) with $k=0$, and suppose that $\lambda_{n}(t)$ can be approximated by a quadratic function of $t$ over any interval of length $\delta t$, which is to say that $\lambda_{n}^{\prime \prime}(t)$ is constant over such an interval. Then the integral in (19) is

$$
\int_{0}^{t} d t^{\prime} e^{i \Omega t^{\prime}} \lambda_{n}^{\prime \prime}\left(t^{\prime}\right)=\delta t \operatorname{sinc}\left(\frac{\Omega \delta t}{2 \pi}\right) \sum_{j=0}^{N-1} e^{i \Omega(j+1 / 2) \delta t} \lambda_{n}^{\prime \prime}(j \delta t),
$$

where $\operatorname{sinc}(x)=\sin (\pi x) /(\pi x), \delta t=t / N$, and $\Omega=\omega-$ $n \omega_{0}$. Take $v=0$, which is the relevant value in following considerations. The sinc factor is strongly concentrated where $|\Omega| \delta t \lesssim 2 \pi$. This is to be contrasted with the total concentration at $\Omega=0$ in the case of a rigid bunch existing for all time. Writing $\delta t=\mu T_{s}$, where $T_{s}$ is the synchrotron period, we may state the concentration condition as a limit on the deviation of the phase velocity from the nominal particle velocity. Since the phase velocity is $\omega / k_{n}$, where $k_{n}=n / R$ is the wave number, we see that for (23) to be appreciable we must have 


$$
\frac{\left|\omega / k_{n}-\beta_{0} c\right|}{\beta_{0} c} \lesssim \frac{\omega_{s}}{\mu n \omega_{0}} .
$$

In the example of Ref. [2] we have $\omega_{s} / \omega_{0}=0.0045$ and the important $n$ are around 700. If the variation of $\lambda_{n}(t)$ can be regarded as quadratic over a hundredth of a synchrotron period, which may be a reasonable guess in view of our simulations to date, then $\mu=0.01$ and the relative deviation of phase velocity from the nominal particle velocity is small compared to 1 at important $n$, being $6.4 \times 10^{-4}$. Since (24) refers to the spectrum of the source, it is only a necessary condition for a particular phase velocity to be involved. For a sufficient condition one requires in addition that the impedance $Z(n, \omega)$ be appreciable in the region where $\omega / k_{n}$ satisfies (24).

Notice that there is an additional mechanism to concentrate the $\omega$ integral near $n \omega_{0}$, in that there is a second order pole in (19) at that point. For higher values of $k$ in (19) there is even more concentration, both because of higher order poles $\Omega^{-(k+2)}$ and because $\lambda_{n}^{(k+2)}$ tends to be constant over bigger intervals $\delta t$, giving sharper peaking of the sinc factor.

Although the above argument is not very precise, it does give a motivation for expanding the impedance in (12) as follows:

$$
Z(n, \omega)=Z\left(n, n \omega_{0}\right)+D_{2} Z\left(n, n \omega_{0}\right)\left(\omega-n \omega_{0}\right)+\cdots,
$$

where $D_{2}$ denotes partial derivative with respect to the second argument of the function. This cannot be done for $n$ such that $n \omega_{0}$ is close to the poles of $Z$ at waveguide cutoffs, $\pm \alpha_{p} c$, which are displayed in (68). We can, however, subtract the pole contribution $Z_{*}$ and expand the remainder:

$$
\begin{aligned}
\tilde{Z}(n, \omega) & =Z(n, \omega)-Z_{*}(n, \omega) \\
& =\tilde{Z}\left(n, n \omega_{0}\right)+D_{2} \tilde{Z}\left(n, n \omega_{0}\right)\left(\omega-n \omega_{0}\right)+\cdots .
\end{aligned}
$$

Both $Z_{*}$ and $\tilde{Z}$ are analytic and bounded for $\operatorname{Im} \omega>v>0$, which implies that the contribution of $Z_{*}$ to (12) can be written either as in (17) or as in (19), and similarly for $\tilde{Z}$. Applying (17) with $Z_{*}$ in place of $Z$, we evaluate the $\omega$ integral by the method of residues to find

$$
\begin{aligned}
& V_{*}(\theta, t)=-\frac{\omega_{0} Q Z_{0} \pi R}{2 \beta_{0} h} \sum_{n \neq 0} e^{i n\left(\theta-\omega_{0} t\right)} \operatorname{sgn}(n) \sum_{p} \Lambda_{p} \int_{0}^{t} d t^{\prime} \lambda_{n}\left(t^{\prime}\right) \\
& \times\left[A(p, n) e^{i A(p, n)\left(t^{\prime}-t\right)}+B(p, n) e^{i B(p, n)\left(t^{\prime}-t\right)}\right] \\
& A(p, n)=\alpha_{p} c-n \omega_{0}, \quad B(p, n)=-\alpha_{p} c-n \omega_{0} .
\end{aligned}
$$

To evaluate the contribution of $\tilde{Z}$ through use of a truncated Taylor expansion (26), we assume conditions
(18) with $m$ equal to the degree of the Taylor polynomial. Putting the Taylor polynomial of $\tilde{Z}$ in place of $Z$ in (19) and choosing $k=m$, we see that the resulting $\omega$ integral converges quadratically. We evaluate the contribution of any monomial $\Omega^{k}$ in the polynomial by using $\mu_{n k}$ in (19). Thus the integral for $\Omega^{k}$ is

$$
\frac{e^{-i n \omega_{0} t}}{2 \pi(-i)^{k+2}} \int_{\operatorname{Im} \Omega=v} d \Omega \frac{e^{-i \Omega t}}{\Omega^{2}} \int_{0}^{t} e^{i \Omega t^{\prime}} \lambda_{n}^{(k+2)}\left(t^{\prime}\right) d t^{\prime} .
$$

At every order $k$ we have concentration of the integral near $\Omega=0$, both from the second order pole and from the $t^{\prime}$ integral in analogy to (23). This provides an heuristic justification for using the Taylor expansion, since at every order the integrand is large only in a neighborhood of the expansion point.

The integral in (29) can be evaluated by pushing the contour to a semicircle at infinity in the lower half-plane. Its value is $-2 \pi i$ times the residue of the second order pole, which is

$$
\left[\frac{d}{d \Omega}\left(e^{-i \Omega t} \int_{0}^{t} e^{i \Omega t^{\prime}} \lambda_{n}^{(k+2)}\left(t^{\prime}\right) d t^{\prime}\right)\right]_{\Omega=0}=-i \lambda_{n}^{(k)}(t) .
$$

Thus the contribution to $V$ from the Taylor polynomial of $\tilde{Z}$ is

$$
\tilde{V}(\theta, t)=\omega_{0} Q \sum_{n} e^{i n\left(\theta-\omega_{0} t\right)} \sum_{k=0}^{m} \frac{1}{k !} D_{2}^{(k)} \tilde{Z}\left(n, n \omega_{0}\right) i^{k} \lambda_{n}^{(k)}(t) .
$$

The first term of the sum contains an alarmingly large piece from $-Z_{*}\left(n, n \omega_{0}\right)$. One is relieved to find that this is canceled by a part of (27), namely, the boundary term that arises when (27) is integrated by parts.

Invoking that cancellation, we find the full approximation to $V$, based on replacing $\tilde{Z}$ by its Taylor polynomial:

$$
\begin{aligned}
V(\theta, t) \approx & 2 \omega_{0} Q \operatorname{Re} \sum_{n=1}^{\infty} e^{i n\left(\theta-\omega_{0} t\right)}\left[Z\left(n, n \omega_{0}\right) \lambda_{n}(t)\right. \\
& +\sum_{k=1}^{m} \frac{1}{k !} D_{2}^{(k)} \tilde{Z}\left(n, n \omega_{0}\right) i^{k} \lambda_{n}^{(k)}(t)-i \frac{Z_{0} \pi R}{2 \beta_{0} h} \\
& \left.\times \sum_{p} \Lambda_{p} \int_{0}^{t} d t^{\prime} \lambda_{n}^{\prime}\left(t^{\prime}\right)\left(e^{i A(p, n)\left(t^{\prime}-t\right)}+e^{i B(p, n)\left(t^{\prime}-t\right)}\right)\right] .
\end{aligned}
$$

We were able to replace the full sum on $n$ by twice the real part of the sum on positive $n$, because the summand $S$ satisfies $S(n)=S(-n)^{*}, S(0)=0$.

The integral in (32) represents retardation effects associated with waveguide cutoffs. It is expected to be largest at those $(p, n)$ for which $A(p, n)=\alpha_{p} c-n \omega_{0}$ is small, giving a primarily reactive effect. The presence of the integral does not add a lot to the cost of a dynamical calculation, since one can store each of the integrals as a matrix 
$M(p, n, t)$, and update that matrix at each time step $\delta t$ by adding the integral from $t$ to $t+\delta t$.

In the numerical work of Refs. [2,3] we retained only the first term of (32). Preliminary numerical work to assess the role of correction terms in (32) was reported in [4], where the full formula with $m=1$ was applied. The added terms seemed to have minor importance, and we take that as justification for neglecting them in [2,3]. A more thorough investigation should be made, however, since the Vlasov integration of [4] did not include a case of fully developed instability.

The reader might have noticed that the terms from the Taylor polynomial of $\tilde{Z}$ can be obtained more directly by putting $\tilde{Z}$ for $Z$ in (12) and invoking the Laplace inversion theorem. The various terms in the polynomial, multiplied by $\hat{\lambda}_{n}(\Omega)$, give Laplace transforms of derivatives of $\lambda_{n}(t)$, provided that enough initial value terms are zero. This calculation gives the same result we have obtained, and the conditions to use the inversion theorem are weaker than (18); namely, for an $m$ th degree polynomial, that $\lambda_{n}$ have only $m+1$ continuous and bounded derivatives, and that $\lambda_{n}^{(k)}(0)=0, k=0, \ldots, m-1$ when $m \geq 1$. Our stronger conditions (18) are needed to justify the Taylor expansion through the argument about concentration of the integral near $\Omega=0$. Concentration from two sources, the second order pole and the $t^{\prime}$ integral, is not obtained under the weaker conditions.
The function $\tilde{Z}(n, \omega)$ is free of poles at $\omega= \pm \alpha_{p} c$, but it does have a logarithmic singularity at those points from $\log \left(\gamma_{p} R\right)$ in (67). Fortunately, the coefficient $U_{p}(n, \omega)$ of the logarithm is exactly the function that displays the shielding cutoff; it and its derivatives with respect to $\omega$ are totally negligible at $\omega=n \omega_{0}=\alpha_{p} c$, a point far below the shielding cutoff for mode $p$ as defined in (73) below. Effectively, $\tilde{Z}(n, \omega)$ behaves as an entire function of $\omega$ near $n \omega_{0}$, and therefore will be well represented by its Taylor polynomial of appropriate degree.

\section{FIELDS IN THE PARALLEL PLATE MODEL}

The longitudinal field for the parallel plate model was derived in the early work of Refs. [6,7], but those papers dealt only with the steady state, effectively finding only the elementary impedance. The elementary impedance for pillbox and toroidal models of the vacuum chamber was found in Ref. [8]. The technique of that paper, which is easily generalized to get the complete impedance, is applied here to the simpler case of perfectly conducting parallel plates. The model could be extended to allow finite conductivity of the plates by using the technique of nonharmonic Fourier series introduced in $[8,9]$.

All field components can be expressed in terms of the Fourier-Laplace transforms of $E_{y}$ and $H_{y}$. The fields may be represented as

$$
\left[\begin{array}{c}
E_{y}(r, \theta, y, t) \\
H_{y}(r, \theta, y, t)
\end{array}\right]=\int_{-\infty}^{\infty} d \omega e^{-i \omega t} \sum_{-\infty}^{\infty} e^{i n \theta} \sum_{p=0}^{\infty}\left[\begin{array}{c}
\cos \left[\alpha_{p}(y+g)\right] \hat{E}_{y n p}(r, \omega) \\
\sin \left[\alpha_{p}(y+g)\right] \hat{H}_{y n p}(r, \omega)
\end{array}\right], \quad \alpha_{p}=\frac{\pi p}{h}
$$

where the $\omega$ integral follows a path $\omega=u+i v$ with some fixed $v>0$. The other fields and the charge/current densities have similar expansions with sine or cosine of $\alpha_{p}(y+g)$ chosen by the following rules:

$$
\begin{aligned}
\left(E_{r}, H_{\theta}, H_{r}, E_{\theta}\right) & \leftrightarrow \text { (sin, cos, cos, sin), } \\
\left(J_{r}, J_{\theta}, J_{y}, \rho\right) & \leftrightarrow \text { (sin, sin, cos, sin). }
\end{aligned}
$$

The Maxwell equations are satisfied term-by-term in the Fourier-Laplace developments, as are the boundary conditions,

$$
H_{y}=0, \quad E_{r}=E_{\theta}=0, \quad y= \pm g .
$$

The Fourier-Laplace amplitudes of $E_{y}, H_{y}$ must satisfy the wave equations

$$
\begin{gathered}
{\left[\frac{1}{r} \frac{\partial}{\partial r}\left(r \frac{\partial}{\partial r}\right)-\left(\Gamma_{p}^{2}+\frac{n^{2}}{r^{2}}\right)\right]\left[\begin{array}{c}
\hat{E}_{y n p} \\
\hat{H}_{y n p}
\end{array}\right]} \\
=\left[\begin{array}{c}
Z_{0}\left[-i(\omega / c) \hat{J}_{y n p}+Q \alpha_{p} c \hat{\rho}_{n p}\right] \\
{\left[-\partial\left(r \hat{J}_{\theta n p}\right) / \partial r+i n \hat{J}_{r n p}\right] / r}
\end{array}\right] \\
\Gamma_{p}^{2}=\alpha_{p}^{2}-(\omega / c)^{2} .
\end{gathered}
$$

$$
Z_{0}=\left(\frac{\mu_{0}}{\epsilon_{0}}\right)^{1 / 2}=120 \pi \Omega(\mathrm{MKS})=\frac{4 \pi}{c}(\mathrm{cgs})
$$

In general, the Laplace transform of the terms of Maxwell's equations with time derivatives would lead to initial value terms, but we set those to zero following the argument in Sec. II before (11). We first solve the equations (36) for real $\omega$ and $\Gamma_{p}^{2}>0$, later performing an analytic continuation to $\operatorname{Im} \omega \geq 0$.

The general solution of (36) is the general solution of the homogeneous equation (a linear combination of modified Bessel functions [10], Sec. 9.6) plus a particular solution of the inhomogeneous equation, thus

$$
\begin{aligned}
& \hat{E}_{y n p}(r)=A_{n p} I_{n}\left(\Gamma_{p} r\right)+B_{n p} K_{n}\left(\Gamma_{p} r\right)+e_{y n p}(r), \\
& \hat{H}_{y n p}(r)=C_{n p} I_{n}\left(\Gamma_{p} r\right)+D_{n p} K_{n}\left(\Gamma_{p} r\right)+h_{y n p}(r) .
\end{aligned}
$$

Given any particular solutions $e_{y n p}, h_{y n p}$, the coefficients $A_{n p}, \ldots, D_{n p}$ must be chosen to satisfy the boundary conditions; namely,

$$
\hat{E}_{y n p}(0)<\infty \text {, }
$$




$$
\lim _{r \rightarrow \infty} \exp \left(\Gamma_{p} r\right) \hat{E}_{y n p}(r)<\infty, \quad \Gamma_{p}>0
$$

and similarly for $\hat{H}_{y n p}$. Here (41) means that below the frequency of the waveguide cutoff the field must die exponentially away from the source.

It is easy to construct particular solutions by the method of variation of parameters, in such a way that those solutions satisfy the boundary conditions by themselves. Then no solution of the homogeneous equations need be added. Specializing to the source for our model, in which $J_{r}=$ $J_{y}=0$, we get the fields meeting all conditions as

$$
\begin{aligned}
& {\left[\begin{array}{l}
\hat{E}_{y n p}(r) \\
\hat{H}_{y n p}(r)
\end{array}\right]} \\
& =\left[K_{n}\left(\Gamma_{p} r\right) \int_{0}^{r} u d u I_{n}\left(\Gamma_{p} u\right)+I_{n}\left(\Gamma_{p} r\right) \int_{r}^{\infty} u d u K_{n}\left(\Gamma_{p} u\right)\right] \\
& \times\left[\begin{array}{c}
-Z_{0} Q \alpha_{p} c \hat{\rho}_{n p}(u) \\
{\left[u \hat{J}_{\theta n p}(u)\right]^{\prime} / u}
\end{array}\right]
\end{aligned}
$$

Noting the value of the Wronskian [10], Sec. 9.6.15,

$$
I_{n}(x) K_{n}^{\prime}(x)-K_{n}(x) I_{n}^{\prime}(x)=-\frac{1}{\pi x}
$$

it is easy to check that (42) satisfies (36). The boundary conditions (40) and (41), are easily verified as well. Because the sources are confined to a finite region of $r$, the second term drops out at large $r$, and the large- $r$ behavior is given by the $K_{n}$, which decreases as $\exp \left(-\Gamma_{p} r\right) r^{-1 / 2}$. At small $r$, the asymptotes $I_{n}(x) \sim$ $(x / 2)^{n} / n !, K_{n}(x) \sim(x / 2)^{-n}(n-1) ! / 2(n \neq 0), K_{0}(x) \sim$ $-\ln x$ show that the fields are bounded at $r=0$, for the type of sources that we consider.

By the Lorentz force law, the longitudinal and transverse forces on the particles are determined by the fields

$$
E_{\theta}, \quad F_{r}=E_{r}+\beta_{0} Z_{0} H_{y}, \quad F_{y}=E_{y}-\beta_{0} Z_{0} H_{r}
$$

In Fourier space the Maxwell equations can be solved algebraically for the corresponding Fourier amplitudes:

$$
\begin{aligned}
& \hat{E}_{\theta n p}=\frac{i}{\Gamma_{p}^{2}}\left[\frac{n \alpha_{p}}{r} \hat{E}_{y n p}+\frac{Z_{0} \omega}{c}\left(\frac{\partial \hat{H}_{y n p}}{\partial r}+\hat{J}_{\theta n p}\right)\right], \\
& \hat{F}_{r n p}=\frac{1}{\Gamma_{p}^{2}}\left[\alpha_{p} \frac{\partial \hat{E}_{y n p}}{\partial r}+\frac{Z_{0} \omega n}{c r} \hat{H}_{y n p}\right]+\beta_{0} Z_{0} \hat{H}_{y n p}, \\
& \hat{F}_{y n p}=\frac{\beta_{0}}{\Gamma_{p}^{2}}\left[\frac{\omega n}{c r} \hat{E}_{y n p}+Z_{0} \alpha_{p}\left(\frac{\partial \hat{H}_{y n p}}{\partial r}+\hat{J}_{\theta n p}\right)\right]+\hat{E}_{y n p} .
\end{aligned}
$$

To compute (42), note that by (1), (34), and (4) we have

$$
\begin{aligned}
\hat{\rho}_{n p} & =\Phi_{n p} \delta(r-R) / R, \quad \hat{J}_{\theta n p}=Q \beta_{0} c \rho_{n p}, \\
\Phi_{n p} & =H_{p} \frac{1}{2 \pi} \int_{0}^{\infty} e^{i\left(\omega-n \omega_{0}\right) t} \lambda_{n}(t) d t, \\
H_{p} & =\frac{1}{g} \int_{-g}^{g} \sin \left[\alpha_{p}(y+g)\right] H(y) d y .
\end{aligned}
$$

Hence, evaluation of (42) gives

$$
\begin{aligned}
\hat{E}_{y n p}(r)= & -Z_{0} Q \alpha_{p} c \Phi_{n p}\left[\theta(r-R) K_{n}\left(\Gamma_{p} r\right) I_{n}\left(\Gamma_{p} R\right)\right. \\
& \left.+\theta(R-r) I_{n}\left(\Gamma_{p} r\right) K_{n}\left(\Gamma_{p} R\right)\right] \\
\hat{H}_{y n p}(r)= & -\Gamma_{p} Q \beta_{0} c \Phi_{n p}\left[\theta(r-R) K_{n}\left(\Gamma_{p} r\right) I_{n}^{\prime}\left(\Gamma_{p} R\right)\right. \\
& \left.+\theta(R-r) I_{n}\left(\Gamma_{p} r\right) K_{n}^{\prime}\left(\Gamma_{p} R\right)\right],
\end{aligned}
$$

where

$$
\theta(x)= \begin{cases}1, & x \geq 0 \\ 0, & x<0\end{cases}
$$

Thus $\hat{E}_{y n p}$ is continuous at $r=R$, but the magnetic field makes a jump that can be computed from (43):

$$
\hat{H}_{y n p}(R+0)-\hat{H}_{y n p}(R-0)=\frac{Q \beta_{0} c \Phi_{n p}}{\pi R} .
$$

Of course, Ampère's law requires a jump at the ribbon beam. There is no discontinuity in $\hat{E}_{\theta n p}$ and $\hat{F}_{y n p}$ since $\partial \hat{H}_{y n p} / \partial r+\hat{J}_{\theta n p}$ is continuous.

Introducing (47) in (45) and evaluating at $r=R \pm 0$ we obtain

$$
\begin{aligned}
\hat{E}_{\theta n p}(R)= & -i Z_{0} Q c \Phi_{n p}\left[\left(\frac{\alpha_{p}}{\Gamma_{p}}\right)^{2} \frac{n}{R} K_{n}\left(\Gamma_{p} R\right) I_{n}\left(\Gamma_{p} R\right)\right. \\
& \left.+\frac{\omega \beta_{0}}{c} K_{n}^{\prime}\left(\Gamma_{p} R\right) I_{n}^{\prime}\left(\Gamma_{p} R\right)\right] \\
\hat{F}_{r n p}(R+0)= & -Z_{0} Q c \Phi_{n p}\left[\frac{\alpha_{p}^{2}}{\Gamma_{p}} K_{n}^{\prime}\left(\Gamma_{p} R\right) I_{n}\left(\Gamma_{p} R\right)\right. \\
& \left.+\left(\beta_{0}+\frac{\omega n}{c R \Gamma_{p}^{2}}\right) \beta_{0} \Gamma_{p} K_{n}\left(\Gamma_{p} R\right) I_{n}^{\prime}\left(\Gamma_{p} R\right)\right]
\end{aligned}
$$

$$
\begin{aligned}
\hat{F}_{y n p}(R)= & -Z_{0} Q c \Phi_{n p}\left[\alpha_{p}\left(1+\frac{\beta_{0} \omega n}{c R \Gamma_{p}^{2}}\right) K_{n}\left(\Gamma_{p} R\right) I_{n}\left(\Gamma_{p} R\right)\right. \\
& \left.+\beta_{0}^{2} \alpha_{p} K_{n}^{\prime}\left(\Gamma_{p} R\right) I_{n}^{\prime}\left(\Gamma_{p} R\right)\right]
\end{aligned}
$$

and

$$
\begin{aligned}
& \hat{F}_{r n p}(R+0)-\hat{F}_{r n p}(R-0) \\
& \quad=\frac{Z_{0} Q c \Phi_{n p}}{\pi R}\left[\left(\frac{\alpha_{p}}{\Gamma_{p}}\right)^{2}-\beta_{0}^{2}-\frac{\beta_{0} \omega n}{c R \Gamma_{p}^{2}}\right] .
\end{aligned}
$$


The longitudinal impedance is defined in terms of the longitudinal field averaged over the transverse distribution, as in Eq. (2). By (34) the Fourier series for $E_{\theta}$ involves $H_{p} \sin \left[\alpha_{p}(y+g)\right]$. We define the dimensionless factor $\Lambda_{p}$ in terms of the $y$ average of that factor, noting (46):

$$
\Lambda_{p}=h H_{p} \int \sin \left[\alpha_{p}(y+g)\right] H(y) d y=2\left(g H_{p}\right)^{2} .
$$

If $H(y)$ is even we have $\Lambda_{p}=0$ for even $p$. For the case of a square step distribution, constant for $y \in[-\delta h / 2, \delta h / 2]$ and zero otherwise, we have

$$
\Lambda_{p}=2 \operatorname{sinc}^{2}(x), \quad x=\frac{p \delta h}{2 h}, \quad p \text { odd }
$$

For a Gaussian distribution with rms width $\sigma_{y} \ll h$ and the $y$ average taken over $\left[-\sigma_{y}, \sigma_{y}\right]$ we have

$$
\Lambda_{p}=2 \operatorname{sinc}(x) e^{-x^{2} / 2}, \quad x=\frac{p \sigma_{y}}{h}, \quad p \text { odd. }
$$

In either case $\Lambda_{p} \approx 2$ for small $p$, which means that there is not much dependence on the vertical size of the beam, since at the values of $n$ of interest only a small number of $p$ modes are unshielded. In the numerical work of [2-4] we chose the square step model.

Now suppose that $(\omega / c)^{2}<\alpha_{1}^{2}$, which is to say that the frequency is below all waveguide cutoffs. Then by (11), (4), and (46), we get the longitudinal impedance from (50) and (54) as

$$
\begin{aligned}
Z(n, \omega)= & \frac{2 \pi i Z_{0} R^{2}}{\beta_{0} h} \sum_{p=1}^{\infty} \Lambda_{p}\left[\left(\frac{\alpha_{p}}{\Gamma_{p}}\right)^{2} \frac{n}{R} I_{n}\left(\Gamma_{p} R\right) K_{n}\left(\Gamma_{p} R\right)\right. \\
& \left.+\frac{\omega \beta_{0}}{c} I_{n}^{\prime}\left(\Gamma_{p} R\right) K_{n}^{\prime}\left(\Gamma_{p} R\right)\right], \quad \omega^{2}<\left(\alpha_{1} c\right)^{2} .
\end{aligned}
$$

Below all cutoffs the impedance is purely imaginary. At higher frequencies the $\Gamma_{p}$ become imaginary one-by-one as the ascending cutoffs are passed, giving the impedance a real part.

In a similar way one can derive horizontal and vertical transverse impedances from the forces (51) and (52). We leave to the reader the appropriate definitions of those impedances, which may depend on the application of interest.

The low frequency limit of the elementary longitudinal impedance can be derived from (57) by the ordinary largeargument expansions of $I_{n}, K_{n}$ ([10], Sec. 9.7.1), supposing that $\pi R / h$ is large compared to 1 . The resulting formula is the same as the corresponding one for the pillbox or rectangular torus model, given in Eq. (4.12) of [8]:

$$
\left.\frac{Z\left(n, n \omega_{0}\right)}{n}\right|_{n=0}=\frac{i Z_{0}}{\beta_{0}} \sum_{p=1}^{\infty} \frac{\Lambda_{p}}{p}\left[\frac{1}{\gamma_{0}^{2}}+\frac{3 \beta_{0}^{2}+1}{8}\left(\frac{h}{\pi p R}\right)^{2}\right] .
$$

For a general complex frequency in the upper half-plane it is convenient to express the impedance in terms of the analytic function

$$
\gamma_{p}(\omega)=\left((\omega / c)^{2}-\alpha_{p}^{2}\right)^{1 / 2}=\left(-\Gamma_{p}^{2}\right)^{1 / 2},
$$

defined in the $\omega$ plane with a cut from $-\alpha_{p} c$ to $\alpha_{p} c$, and positive for $\omega>\alpha_{p} c$. With this definition we have

$$
\gamma_{p}(-\omega)=-\gamma_{p}(\omega), \quad|\omega|>\alpha_{p} c,
$$

and the boundary values on the cut satisfy

$$
\gamma_{p}(u+i 0)=i\left|\gamma_{p}(u+i 0)\right|=i \Gamma_{p}(u), \quad-\alpha_{p} c<u<\alpha_{p} c .
$$

Now recall the following relations that hold for $-\pi<$ $\arg x \leq \pi / 2$ [10], Secs. 9.6.3, 9.6.4:

$$
\begin{aligned}
I_{n}(x) & =i^{-n} J_{n}(i x), \\
K_{n}(x) & =(\pi / 2) i^{n+1}\left[J_{n}(i x)+i Y_{n}(i x)\right],
\end{aligned}
$$

where $J_{n}$ and $Y_{n}$ are Bessel functions of the first and second kinds, respectively, [10], Sec. 9.1. Substituting (62) in (57) and applying (61) we find

$$
\begin{aligned}
Z(n, \omega)= & Z_{0} \frac{(\pi R)^{2}}{\beta_{0} h} \sum_{p=1}^{\infty} \Lambda_{p}\left[\frac{\omega \beta_{0}}{c} J_{|n|}^{\prime}\left(\gamma_{p} R\right) H_{|n|}^{(1) \prime}\left(\gamma_{p} R\right)\right. \\
& \left.+\left(\frac{\alpha_{p}}{\gamma_{p}}\right)^{2} \frac{n}{R} J_{|n|}\left(\gamma_{p} R\right) H_{|n|}^{(1)}\left(\gamma_{p} R\right)\right], \quad \operatorname{Im} \omega>0 .
\end{aligned}
$$

Here and in the following we invoke the Hankel functions $H_{n}^{(1,2)}(x)=J_{n}(x) \pm i Y_{n}(x)$. We have used Bessel function behavior under $n \rightarrow-n$ ([10], Sec. 9.1.5), to state (63) in a form correct and convenient for either sign of $n$.

Since $J_{n}(z)$ is an entire function of $z$, and $Y_{n}(z)$ is analytic in the $z$ plane with a cut along the negative real axis $[10,11]$, we see from $(63)$ that $Z(n, \omega)$ is analytic in the upper half $\omega$ plane at fixed integer $n$. To describe and study the singularities of $Z$ on the real $\omega$ axis, it is useful to define the functions

$$
\begin{aligned}
U_{p}(n, \omega)= & \frac{\omega \beta_{0}}{c} J_{|n|}^{\prime 2}\left(\gamma_{p} R\right)+\left(\frac{\alpha_{p}}{\gamma_{p}}\right)^{2} \frac{n}{R} J_{|n|}^{2}\left(\gamma_{p} R\right), \\
V_{p}(n, \omega)= & \frac{\omega \beta_{0}}{c} J_{|n|}^{\prime}\left(\gamma_{p} R\right) Y_{|n|}^{\prime}\left(\gamma_{p} R\right) \\
& +\left(\frac{\alpha_{p}}{\gamma_{p}}\right)^{2} \frac{n}{R} J_{|n|}\left(\gamma_{p} R\right) Y_{|n|}\left(\gamma_{p} R\right),
\end{aligned}
$$

thus

$$
Z(n, \omega)=Z_{0} \frac{(\pi R)^{2}}{\beta_{0} h} \sum_{p=1}^{\infty} \Lambda_{p}\left[U_{p}(n, \omega)+i V_{p}(n, \omega)\right] .
$$

Referring to the expressions of $J_{n}$ and $Y_{n}$ by power series ([10], Secs. 9.1.10, 9.1.11), we see that $U_{p}$ involves only 
even powers of $\gamma_{p}$, and represents an entire function of $\omega$. On the other hand, $V_{p}$ involves poles and branch points on the real axis where $\gamma_{p}=0$. By [10], Sec. 9.1.11, the functions $Y_{n}(z)-(2 / \pi) \ln (z / 2) J_{n}(z)$ and $Y_{n}^{\prime}(z)-(2 / \pi) \times$ $\ln (z / 2) J_{n}^{\prime}(z)$ are meromorphic (analytic except for poles) in the whole $z$ plane. Consequently, it is useful to rewrite $V_{p}$ as $V_{p}=V_{p}^{(1)}+V_{p}^{(2)}$, where

$$
\begin{gathered}
V_{p}^{(1)}=V_{p}-\frac{2}{\pi} \ln \left(\frac{\gamma_{p} R}{2}\right) U_{p}, \\
V_{p}^{(2)}=\frac{2}{\pi} \ln \left(\frac{\gamma_{p} R}{2}\right) U_{p} .
\end{gathered}
$$

Now $V_{p}^{(1)}(n, \omega)$, involving only even powers of $\gamma_{p}$, is meromorphic in $\omega$, whereas $V_{p}^{(2)}(n, \omega)$ is an entire function of $\omega$ times the logarithmic factor that is analytic in the $\omega$ plane with branch cut $\left[-\alpha_{p} c, \alpha_{p} c\right]$. The poles of $V_{p}^{(1)}$ come from $\gamma_{p}^{-2}$ and $J_{|n|}^{\prime} Y_{|n|}^{\prime}$, except for $n=0$, in which case there is no pole. The poles alone make the following contribution to the impedance:

$$
\begin{aligned}
Z_{*}(n, \omega)= & i \operatorname{sgn}(n)\left(1-\delta_{0 n}\right) \frac{Z_{0} \pi R}{2 \beta_{0} h} \sum_{p} \Lambda_{p}\left[\frac{n \omega_{0}-\alpha_{p} c}{\omega-\alpha_{p} c}\right. \\
& \left.+\frac{n \omega_{0}+\alpha_{p} c}{\omega+\alpha_{p} c}\right],
\end{aligned}
$$

where $\operatorname{sgn}(n)$ is the sign of $n$. The pole location $\omega= \pm \alpha_{p} c$ is the $p$ th waveguide cutoff for the parallel plate system. Below the cutoff frequency the $p$ th mode is "evanescent" (spatially localized), then at cutoff turns into a propagating wave, allowing energy to radiate to infinity. Correspondingly, this is also the point at which $U_{p}+i V_{p}$ first acquires a real part, as the frequency is increased from zero. As we shall see, the poles are associated with retardation effects in the deforming bunch formalism.

The poles do not show up as infinities or even sharp peaks in $Z(n)=Z\left(n, n \omega_{0}\right)$, since $Z_{*}\left(n, n \omega_{0}\right)=$ $i \operatorname{sgn}(n) Z_{0}\left(\pi R / \beta_{0} h\right) \sum_{p} \Lambda_{p}$ is bounded and independent of $n$ except for a sign. Consequently, it is likely that the poles have not been noticed by previous investigators, who studied mostly $Z(n)$.

In Sec. II we encountered the question of the asymptotic behavior of $Z(n, u+i v), u \rightarrow \pm \infty, v>0$. Let us first use the reflection properties of Bessel functions ([10], Secs. 9.1.35, 9.1.39) and Eq. (60) to write the impedance in a form convenient to show its behavior at $u=-\infty$. We have

$$
\begin{aligned}
Z(n,-\omega)= & Z_{0} \frac{(\pi R)^{2}}{\beta_{0} h} \sum_{p=1}^{\infty} \Lambda_{p}\left[\frac{\omega \beta_{0}}{c} J_{|n|}^{\prime}\left(\gamma_{p} R\right) H_{|n|}^{(2) \prime}\left(\gamma_{p} R\right)\right. \\
& \left.-\left(\frac{\alpha_{p}}{\gamma_{p}}\right)^{2} \frac{n}{R} J_{|n|}\left(\gamma_{p} R\right) H_{|n|}^{(2)}\left(\gamma_{p} R\right)\right]
\end{aligned}
$$

where on the right-hand side $\gamma_{p}=\gamma_{p}(\omega)$. As a check of
(69) one may verify the reality property $Z(n, u)=$ $Z(-n,-u)^{*}$ using (63), (60), and (61). Now evaluate (63) at $\omega=u+i v$ and (69) at $\omega=u-i v$, and apply the large-argument asymptotic forms of Bessel functions ([10], Sec. 9.2). The result is

$$
Z(n, u+i v) \sim \frac{Z_{0} \pi R}{h}\left[1-e^{2 i u R / c}\right] \sum_{p} \Lambda_{p}, \quad u \rightarrow \pm \infty .
$$

Now we see that the $\omega$ integral of the first term in the square bracket of (17) exists since it is understood as the symmetric limit of (10), the constant term in $Z$ being the same in the two limits of (70). The integral with factor $\exp (i u R / c)$ converges by virtue of oscillations, without the benefit of asymptotic cancellations.

In deriving (70) we have invoked uniform convergence of the $p$ series with respect to $u$ to justify taking the limit under the sum. Such convergence holds for the Gaussian model of the vertical distribution, as is seen from (56) and the integral representation ([12], Sec. III.14.19a)

$$
\begin{aligned}
H_{n}^{(1)}(z)= & {\left[\frac{2}{\pi z}\right]^{1 / 2} \frac{e^{i(z-\pi n / 2-\pi / 4)}}{\Gamma(n+1 / 2)} } \\
& \times \int_{0}^{\infty} e^{-t} t^{n-1 / 2}\left[1+\frac{i t}{2 z}\right]^{n-1 / 2} d t .
\end{aligned}
$$

The corresponding formula for $H_{n}^{(2)}(z)$ is obtained by changing $i$ to $-i$ in (71), and an integral representation for $J_{n}$ is given by $J_{n}=\left[H_{n}^{(1)}+H_{n}^{(2)}\right] / 2$. Using these results and remembering that $\gamma_{p}$ never vanishes because $v>0$, one can show that the coefficient of $\Lambda_{p}$ in the $p$ sum of (63) or (69) has a bound of the form $C p^{2}$, where $C$ is a constant, independent of $u$. By (56) and the Weierstrass $M$ test, the $p$ sum converges uniformly in $u$. A similar result holds for any vertical distribution that is sufficiently smooth to ensure that $\sum_{p} p^{2}\left|\Lambda_{p}\right|$ converges.

In a similar way we can find the asymptotic behavior of $Z(n, \omega)$ as $\omega$ tends to infinity along any direction in the upper half-plane. To get the behavior in the first quadrant put $\omega=i v+\rho \exp (i \phi), 0 \leq \phi \leq \pi / 2$ in the right-hand side of (63) and let $\rho \rightarrow \infty$ at fixed $\phi$. For behavior in the second quadrant do the same in (69), but with $-\pi / 2 \leq$ $\phi \leq 0$. Thus we find that $Z$ is bounded at infinity in the upper half-plane, a result that we require in closing contours at infinity.

For further analysis we require a method to evaluate the Bessel functions. Since the important values of $n$ are quite large, asymptotic expansions for large $n$ are essential. We are mainly interested in $\omega$ close to $n \omega_{0}$, so we consider evaluation of $J_{n}(n z), Y_{n}(n z)$ and the corresponding differentiated functions, where

$$
n z=\gamma_{p}\left(n \omega_{0}\right) R=n\left[\beta_{0}^{2}-\left(\frac{\pi p R}{n h}\right)^{2}\right]^{1 / 2} .
$$

The transition from exponential to oscillatory behavior of 
the Bessel functions occurs near $z=1$, with exponential decrease of $J_{n}(n z)$ and increase of $Y_{n}(n z)$ as $z$ is decreased below transition, and similarly for the derivatives. It is then reasonable to define the shielding cutoff $n_{0}(p)$ for the $p$ th mode, and the corresponding $z$, as follows (assuming $\beta_{0} \approx$ 1):

$$
n_{0}(p)=\pi p\left[\frac{R}{h}\right]^{3 / 2}, \quad z=\left[\beta_{0}^{2}-\left(\frac{h}{R}\right)^{1 / 2}\right]^{1 / 2} .
$$

This generalizes our previous shielding cutoff [2], which was for $p=1$, and ensures that $U_{p}\left(n, n \omega_{0}\right)$ first becomes appreciable on increasing $n$ at roughly $n=n_{0}(p)$.

Since the behavior of the Bessel functions changes rapidly near $z=1$, it is convenient when working in that region to use expansions that are accurate at large $n$ uniformly in $z$. We use the Olver expansions ([10], Secs. 9.3.35-9.3.46) which are expressed in terms of Airy functions and are uniform in $z$ to a remarkable extent, in the entire sector $|\arg z| \leq \pi-\epsilon$, any $\epsilon>0$. Using results of Decker [13], we get a fast evaluation of the Olver expansions, as explained in [8].

For $z$ a bit less than 1 the function $U_{p}(n, \omega)$ of (65) becomes negligible, but we still have to evaluate $V_{p}(n, \omega)$. For that it is sufficient to use the simpler Debye expansions [14]. Instead of evaluating the individual Bessel functions and then combining their values numerically, it is essential first to make an analytic reduction of $V_{p}$ which accounts for close cancellations of exponential factors (very large or very small in this region). This was done in [14] for the torus impedance. To retrieve the parallel plate case from Eq. (2.19) of [14], put $\sigma_{n p}^{(M, E)}=0$. Also note that the definition of $\Lambda_{p}$ in this paper is twice that of [14].

In practice we make the change from Olver to Debye expansions at about $z=0.9$. We take enough terms in the $p$ expansion to give a negligible remainder, but in many cases the first term alone gives a fairly good estimate, especially in the important region near the first shielding cutoff. Evaluating the first term, $p=1$, by the leading term in the Olver expansion, we get the result (for $\beta_{0}=1$ ) given in Ref. [15],

$$
\frac{\operatorname{Re} Z(n)}{n}=2 Z_{0}\left[\frac{\pi R}{h n}\right]^{2} \exp \left[-\frac{2}{3 n^{2}}\left(\frac{\pi R}{h}\right)^{3}\right] .
$$

The results mentioned in connection with Eq. (2) of Ref. [2] can be read off from this formula. The exact evaluation for Fig. 1 of [2] included all modes up to $p=$ 43, but the higher $p$ affect mainly the behavior at large $n$.

\section{SUMMARY AND OUTLOOK}

We have shown how to extend the impedance formalism to account correctly for a beam with evolving charge density. This was done for a particular model in which the particles move on circular orbits between conducting parallel plates. The basic technique was to make a
Fourier-Laplace transform of the Maxwell equations and the charge/current source, then make a detailed study of the analyticity of the solution in the complex frequency $\omega$. It will be interesting to study other soluble models in a similar way, for instance, that in which the parallel plates are replaced by a closed pillbox. Models including electrical resistance of the vacuum chamber, with or without trajectory curvature, are also of interest.

The mathematics of this work is interesting and much more subtle than we expected at the outset. One has to be quite careful because of surprising cancellations and convergence questions. We were able to give reasonable assumptions on the charge density so that most of our conclusions follow rigorously, except that we have no analytic estimate of accuracy of the approximations displayed in Eq. (32). The first approximation has been the mainstay of simulations to date. More work will be needed to clarify the quantitative importance and physical meaning of higher terms, especially the integral in (32) which arose from poles at waveguide cutoffs.

The transverse part of the CSR force has usually been ignored in dynamical simulations, on the belief that its electric and magnetic components nearly cancel for relativistic velocities. It would be worthwhile to test this assumption concretely in a simulation, using the expressions for the transverse force given in Sec. IV.

A complementary approach to the CSR force is to work directly in space-time, rather than with integral transforms. This was done for the same physical model by Murphy, Krinsky, and Gluckstern [16]. Their analysis does not deal with our questions of retardation with bunch deformation, since it deals with the steady state case and just results in a wake function, approximately the Fourier transform of our elementary impedance. In the time-dependent case with evolving bunch one can write down the exact force as an integral over the space-time Green function of the system. Evaluation of this integral would be too expensive, but one could look for approximations analogous to those that we derived by integral transforms. That is another interesting topic for further research.

\section{ACKNOWLEDGMENTS}

Our research was supported in part by Department of Energy Contracts No. DE-AC03-76SF00515, No. DEAC03-76SF00098, and No. DE-FG03-99ER41104.

[1] B. W. Zotter and S. Kheifets, Impedances and Wakes in High Energy Particle Accelerators (World Scientific, Singapore, 1998).

[2] M. Venturini, R. Warnock, R. Ruth, and J.A. Ellison, Phys. Rev. ST Accel. Beams 8, 014202 (2005). 
[3] M. Venturini and R. Warnock, Phys. Rev. Lett. 89, 224802 (2002).

[4] R. Warnock and M. Venturini, in Proceedings of the Particle Accelerator Conference, Portland, OR, 2003 (IEEE, Piscatway, NJ, 2003), p. 3144, paper RPPB061.

[5] G. B. Folland, Fourier Analysis and Its Applications (Wadsworth and Brooks/Cole, Pacific Grove, CA, 1992).

[6] J. Schwinger, "On Radiation by Electrons in a Betatron," unpublished report, 1945, transcribed in Lawrence Berkeley National Laboratory Report No. LBL-39088, 1996.

[7] J. Nodvick and D. Saxon, Phys. Rev. 96, 180 (1954).

[8] R. Warnock and P. Morton, Part. Accel. 25, 113 (1990).

[9] R. L. Warnock, G. R. Bart, and S. Fenster, Part. Accel. 12, 179 (1982).

[10] Handbook of Mathematical Functions, edited by M. Abramowitz and I. A. Stegun, Applied Mathematics Series Vol. 55 (National Bureau of Standards, Washington, DC, 1972).
[11] Higher Transcendental Functions, edited by A. Erdélyi et al., Bateman Manuscript Project Vol. II (McGraw-Hill, New York, 1953).

[12] A.F. Nikiforov and V.B. Uvarov, Special Functions of Mathematical Physics (Birkhäuser, Basel, 1988). Note that in Section III.15, the factor $z^{\nu}$ appears erroneously in Eqs. (19a) and (20a), whereas it appears correctly in Eqs. (19) and (20). Note also that $\operatorname{Re} z>0$ is implied in (19) and (20), whereas the change of variable and rotation of direction of integration to obtain (19a) and (20a) accomplishes an analytic continuation that relaxes that condition.

[13] G. A. Decker, Ph.D. Thesis, Cornell University, 1986.

[14] K.-Y. Ng and R. Warnock, Phys. Rev. D 40, 231 (1989). Note the following corrections: in the sentence following Eq. (3.12) omit "large"; in Eq. (3.16) replace the initial minus sign by plus.

[15] R. Warnock, in Proceedings of the IEEE Particle Accelerator Conference, San Francisco, CA, 1991 (IEEE, Piscataway, NJ, 1991), p. 1824.

[16] J. Murphy, S. Krinsky, and R. Gluckstern, Part. Accel. 57, 9 (1997). 